\title{
Identificación de factores demográficos y académicos que inciden en la aprobación de la asignatura Fisiología en la carrera de Medicina de la Universidad de Ciencias Médicas
}

\author{
Identifying Demographic and Academic Issues that Influence the Passing or Failing of \\ the Physiology Course in the Medicine Study Program of UCIMED \\ (University of Medical Sciences)
}

\author{
Juan Carlos Vanegas-Pissa ${ }^{1}$ \\ Universidad de Ciencias Médicas \\ San José, Costa Rica \\ vanegaspj@ucimed.com \\ Hilda Sancho-Ugalde ${ }^{2}$ \\ Universidad de Ciencias Médicas \\ San José, Costa Rica \\ sanchouh@ucimed.com
}

Recibido 5 de mayo de 2014 • Corregido 28 de enero de 2015 • Aceptado 27 de abril de 2015

\begin{abstract}
Resumen. En este artículo, derivado de un trabajo de investigación, que analiza algunos factores académicos y demográficos que podrían influir en la aprobación o reprobación de la materia de Fisiología en la carrera de Licenciatura en Medicina y Cirugía, impartida en la Universidad de Ciencias Médicas, en el periodo 2008 al 2011. Este estudio es de tipo longitudinal-retrospectivo. Se analizan las calificaciones obtenidas por el estudiantado de la UCIMED que inscribieron la asignatura de Fisiología, por primera, segunda o tercera ocasión, durante el periodo de la investigación, los semestres en que fueron obtenidas, quienes aprobaron o reprobaron, las características sociodemográficas, otras asignaturas aprobadas o reprobadas previamente y sus notas. Para el análisis de los datos se utilizó el Software Stata 13 (Data Analysis and Statistical Software), se estima un modelo de regresión logística, para determinar las variables que explican la aprobación de la asignatura de Fisiología. Los resultados indican que las variables que mayor efecto tienen sobre la probabilidad de aprobar o no la asignatura de Fisiología son: la calificación en las asignaturas de Anatomía I y de Histología.
\end{abstract}

Palabras claves. Aprobación, reprobación, fisiología, medicina, regresión logística

\footnotetext{
${ }^{1}$ Maestría Académica en Estadística por la Universidad de Costa Rica. Egresado del Programa en Bioestadística de la Universidad de Chile. Licenciado en Matemáticas y Física por la Universidad de los Llanos, Colombia. Profesor de la UCIMED. Actualmente es el Estadístico de la UCIMED.

${ }^{2}$ Maestría Académica en Ciencias Biomédicas la Universidad Autónoma de México. Actualmente es la Directora de la Unidad de Aseguramiento de la calidad académica de la UCIMED.
} 
doi: http://dx.doi.org/10.15359/ree.19-2.5

URL: http://www.una.ac.cr/educare

CORREO: educare@una.cr

\begin{abstract}
This paper, product of a research project, analyzes some academic and demographic issues that might influence students passing or failing Physiology in the Licentiate Study Program in Medicine and Surgery at UCIMED (University of Medical Sciences) between 2008 and 2011. This was a retrospective cohort study. We analyzed the grades obtained by the students who were taking Physiology for the first, second or third time during the research period, the semesters in which the grades were obtained, who passed or failed the course, their sociodemographic characteristics, and other courses passed or failed previously with their corresponding grades. For the data analysis, we used the Stata 13 software (Data Analysis and Statistical Software) with a logistic regression model to determine the variables, which explain the passing or failing of the Physiology course. The results showed that the variables with a greater effect on the probability of passing or not the Physiology course are the grades obtained in Anatomy I and Histology.
\end{abstract}

Keywords. Passing, Failing, Physiology, Medicine, Logistic regression.

La deserción de las carreras de Medicina y Cirugía constituye un tema que ha sido objeto de atención y análisis, en la búsqueda de las causas prevenibles de esta. El abandono, debido a la reprobación de cursos, es frecuente durante los primeros semestres y aunque las causas son complejas, los aspectos académicos relacionados con las materias de esos niveles no escapan al análisis y es allí donde se ubica, generalmente, el curso Fisiología humana. Varios estudios han abordado este tema, analizando variables tales como los procesos de socialización demandados por el entorno universitario, el nivel de los estudios y los aspectos académicos y éticos (Ferguson, James, y Madeley, 2002; Hernández-Mata, Hernández-Castro, Nieto-Caraveo y Hernández-Sierra, 2005; Ramírez, 2012).

Todo el análisis se ha hecho con el objetivo de delinear las políticas, los mecanismos y las acciones que pueden ser instrumentados para disminuir los problemas que los generan y que vulneran la calidad de la educación superior, en la escogencia de las personas aspirantes de primer ingreso, su transcurso en la carrera y la eficiencia terminal de la institución (Vélez y Roa, 2005).

El curso de Fisiología Humana tiene características académicas y de aprendizaje que lo ubican como uno de los de mayor complejidad dentro de los requeridos para la formación de profesionales del área de la salud. La formación previa en las ciencias básicas puras es fundamental para acceder a los conocimientos fisiológicos. El desarrollo y aplicación de las capacidades intelectuales de mayor complejidad son requeridas para el desarrollo del pensamiento lógico, sintético y evaluador, necesario para la aprehensión de los conocimientos propios de esta ciencia. Los altos índices de reprobación en diferentes asignaturas, propias de los semestres iniciales del plan de estudios, han conducido a realizar diversas actividades institucionales con el objetivo de incrementar la eficiencia terminal y detectar las causas prevenibles, mediante el control y la evaluación periódica de aquel alumnado que presente un rendimiento académico menor al que se espera. Como parte de esa evaluación se realiza este estudio. 
doi: http://dx.doi.org/10.15359/ree.19-2.5

URL: http://www.una.ac.cr/educare

En cualquier nivel de educación, uno de los principales problemas por afrontar de las instituciones es la reprobación que se presenta en cualquier asignatura, a pesar de existir un sinnúmero de estudios al respecto con diversos abordajes. La reducción de la reprobación no es sencilla, debido a que esta problemática es multifactorial, porque aborda razones de tipo económico, político, social, familiar, psicológico y pedagógico, entre otras (Romo y Fresán, 2001).

\section{Metodología}

La población en estudio fue el total de estudiantes de la carrera de Medicina que matricularon la asignatura de Fisiología en el periodo 2008 al 2011. La información proviene de la base de datos de la UCIMED y se utilizó, como criterio de inclusión, haber cursado todas las asignaturas con alta reprobación que preceden a la asignatura de Fisiología; de acuerdo con las estadísticas de la UCIMED, estas asignaturas son: Química general, Biología y laboratorio, Fundamentos de química orgánica, Física para ciencias médicas, Anatomía I, Anatomía II, Histología y Embriología; obteniéndose una muestra de 256 estudiantes, la cual, para este estudio y los análisis respectivos, se supondrá como aleatoria. Este estudio es de tipo no experimental, exploratorio, retrospectivo, longitudinal y analítico.

La nota obtenida en la asignatura de Fisiología (AprobaM6), recodificada como aprobada (70 o más) o reprobada, menos de 70 en una escala de 0 a 100, la cual fungirá como variable dependiente.

Como variables académicas predictoras, se consideraron las calificaciones obtenidas en una escala de 0 a 100, en las asignaturas del primer al tercer semestre con alta reprobación histórica y la primera vez que la matricularon; es decir, no se tuvieron en cuenta las notas de las reprobaciones, estas variables son:

1. Nota obtenida en la asignatura de Química general (NotaCB1): Corresponde a la calificación obtenida en la asignatura CB1, la primera vez que la matriculó, entre los años 2008 a 2011, en el primero o segundo periodo académico.

2. Número de veces que matriculó la asignatura de Química general (NVMCB1): Corresponde al número de veces que matriculó esta asignatura; es decir, no la repitió y la repitió al menos una vez, entre los años 2008 a 2011, en el primero o segundo periodo académico.

3. Nota obtenida en la asignatura de Biología y laboratorio (NotaCB2): Corresponde a la calificación obtenida en la asignatura CB2, la primera vez que la matriculó, entre los años 2008 a 2011, en el primero o segundo periodo académico.

4. Número de veces que matriculó la asignatura de Biología y laboratorio (NVMCB2): Corresponde al número de veces que matriculó esta asignatura; es decir, no la repitió y la repitió al menos una vez, entre los años 2008 a 2011, en el primero o segundo periodo académico. 
5. Nota obtenida en la asignatura de Química orgánica (NotaCB7): Corresponde a la calificación obtenida en la asignatura CB7, la primera vez que la matriculó, entre los años 2008 a 2011, en el primero o segundo periodo académico.

6. Número de veces que matriculó la asignatura de Química orgánica (NVMCB7): Corresponde al número de veces que matriculó esta asignatura; es decir, no la repitió y la repitió al menos una vez, entre los años 2008 a 2011, en el primero o segundo periodo académico.

7. Nota obtenida en la asignatura de Física para ciencias médicas (NotaCB9): Corresponde a la calificación obtenida en la asignatura CB9, la primera vez que la matriculó, entre los años 2008 a 2011, en el primero o segundo periodo académico.

8. Número de veces que matriculó la asignatura de Física para ciencias médicas (NVMCB9): Corresponde al número de veces que matriculó esta asignatura; es decir, no la repitió y la repitió al menos una vez, entre los años 2008 a 2011, en el primero o segundo periodo académico.

9. Nota obtenida en la asignatura de Anatomía I (NotaM2): Corresponde a la calificación obtenida en la asignatura M2, la primera vez que la matriculó, entre los años 2008 a 2011 , en el primero o segundo periodo académico.

10. Número de veces que matriculó la asignatura de Anatomía I (NVMM2): Corresponde al número de veces que matriculó esta asignatura; es decir, no la repitió y la repitió al menos una vez, entre los años 2008 a 2011, en el primero o segundo periodo académico.

11. Nota obtenida en la asignatura de Anatomía II (NotaM3): Corresponde a la calificación obtenida en la asignatura M3, la primera vez que la matriculó, entre los años 2008 a 2011, en el primero o segundo periodo académico.

12. Número de veces que matriculó la asignatura de Anatomía II (NVMM3): Corresponde al número de veces que matriculó esta asignatura; es decir, no la repitió y la repitió al menos una vez, entre los años 2008 a 2011, en el primero o segundo periodo académico.

13. Nota obtenida en la asignatura de Histología (NotaM4): Corresponde a la calificación obtenida en la asignatura M4, la primera vez que la matriculó, entre los años 2008 a 2011 , en el primero o segundo periodo académico.

14. Número de veces que matriculó la asignatura de Histología (NVMM4): Corresponde al número de veces que matriculó esta asignatura; es decir, no la repitió y la repitió al menos una vez, entre los años 2008 a 2011, en el primero o segundo periodo académico.

15. Nota obtenida en la asignatura de Embriología (NotaM5): Corresponde a la calificación obtenida en la asignatura M5, la primera vez que la matriculó, entre los años 2008 a 2011, en el primero o segundo periodo académico.

16. Número de veces que matriculó la asignatura de Embriología (NVMM5): Corresponde al número de veces que matriculó esta asignatura; es decir, no la repitió y la repitió al menos una vez, entre los años 2008 a 2011, en el primero o segundo periodo académico. 
doi: http://dx.doi.org/10.15359/ree.19-2.5

URL: http://www.una.ac.cr/educare

También se tomaron como variables académicas predictoras, el número de veces que repitió cualquiera de las asignaturas antes mencionadas; es decir, no la repitió y la repitióal menos una vez. Cabe señalar que no se consideraron estudiantes que convalidaron, equipararon, se retiraron, presentaron exámenes por suficiencia de al menos una de las anteriores asignaturas.

Dentro de las variables socio-demográficas se tomaron en cuenta las siguientes:

- Año de ingreso: Corresponde al año de ingreso entre los años 2008 al 2011.

- Período de ingreso: Corresponde a si ingresó en el primer semestre del año o en el segundo semestre del año.

- Edad que tenía cuando matriculó la asignatura de Fisiología

- Sexo: Masculino o femenino

- Nacionalidad: Costarricense y no costarricense

- Región: Provincias ubicadas en gran parte en el Valle Central (San José, Alajuela, Cartago y Heredia) y las de fuera del Valle Central (Guanacaste, Puntarenas y Limón)

Con estas variables se ajustó un modelo de regresión logística, con el objeto de determinar las variables predictoras que son factores de riesgo o factores protectores que puedan explicar la aprobación o no de la asignatura de Fisiología. Para el análisis de los datos se utilizó el software Stata 13 (Data Analysis and Statistical Software).

Se realizaron las siguientes pruebas diagnósticas para determinar la validez del modelo estimado:

- La verosimilitud del modelo $(p=0.000)$

- Las pruebas de bondad de ajuste chi-cuadrado y la Hosmer-Lemeshow, con valores de $p=0.3760$ y $p=0.3955$ respectivamente.

- La capacidad predictiva del modelo, con la medida Adj Count $\mathrm{R}^{2}=0.6745$

\section{Resultados}

De los 586 casos de la muestra, que ingresaron entre los años 2008 hasta el 2011 en el primero o segundo periodo académico, $40.4 \%$ fueron hombres. Del total de la población, 90.8\% fueron costarricenses y de ellos el $93.7 \%$ provino de alguna provincia del Valle Central. El promedio de edad fue de $19.5 \pm 1.4$ años y, el 75\%: estudiantes en estudio tenían 20 años o menos cuando matricularon la asignatura de Fisiología.

Para la estimación del modelo, se excluyeron las variables que presentaron colinealidad y se utilizó el procedimiento de regresión stepwise (paso a paso) para seleccionar el mejor modelo, obteniéndose el modelo 1: 
doi: http://dx.doi.org/10.15359/ree.19-2.5

URL: http://www.una.ac.cr/educare

CORREO: educare@una.cr

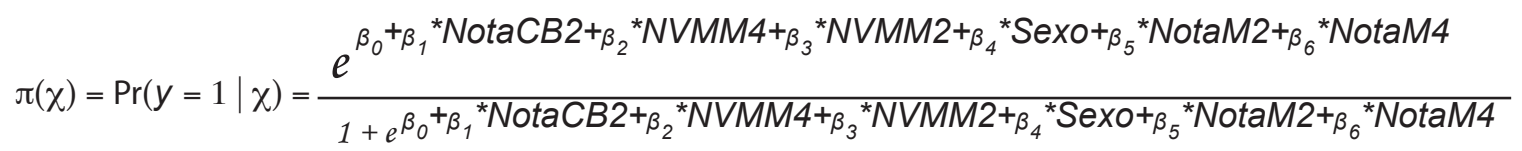

Las variables analizadas que explican la aprobación o reprobación de la asignatura de Fisiología en la carrera de Medicina de la UCIMED fueron:

- Sexo

- Nota obtenida en la asignatura de Biología y laboratorio

- Nota obtenida en la asignatura de Anatomía I

- Número de veces que matriculó la asignatura de Anatomía I

- Nota obtenida en la asignatura de Histología

- Número de veces que matriculó la asignatura de Histología

En la tabla 1, se observa que las variables, "número de veces que matriculó las asignaturas de "Histología" y "Anatomía I" (NVMM4 y NVMM2 respectivamente) son las que presentan mayores Odds Ratio (razón de probabilidades); es decir, el no repetir estas asignaturas aumenta la razón de aprobar la asignatura de Fisiología en 2.34 y 2.85 veces respectivamente en relación al que la repite al menos una vez.

Tabla 1

Influencia de las variables independientes en la probabilidad de aprobar la asignatura de Fisiología. UCIMED 2008-2011. $(n=256)$

\begin{tabular}{lcccccc}
\hline AprobM6 & Odds Ratio & Std. Err. & $\mathrm{z}$ & \multicolumn{2}{c}{$\mathrm{P}>|\mathrm{z}|$} & \multicolumn{2}{c}{ 95\% Conf. Interval } \\
\hline NotaCB2 & 1.081403 & 0.0185094 & -4.57 & $0.000^{*}$ & 1.045727 & 1.118296 \\
NVMM4 & 2.345317 & 0.9570107 & -2.09 & $0.037^{*}$ & 1.054065 & 5.218382 \\
NVMM2 & 2.849047 & 1.074 .293 & -2.78 & $0.005^{*}$ & 1.360618 & 5.965723 \\
Sexo & 1.730538 & 0.3476808 & -2.73 & $0.006^{*}$ & 1.167259 & 2.565638 \\
NotaM2 & 1.063775 & 0.0151796 & -4.33 & $0.000^{*}$ & 1.034436 & 1.093946 \\
NotaM4 & 1.087642 & 0.0185769 & -4.92 & $0.000^{*}$ & 1.051843 & 1.124668 \\
Constante & $1.89 \mathrm{E}-08$ & $3.60 \mathrm{E}-08$ & -9.32 & 0.000 & $4.49 \mathrm{E}-10$ & $7.94 \mathrm{E}-07$ \\
\hline
\end{tabular}

Nota: Elaboración propia. *Odds Ratio significativos al $5 \%$. 
doi: http://dx.doi.org/10.15359/ree.19-2.5

URL: http://www.una.ac.cr/educare

Para analizar las variables que tienen mayor efecto sobre la posibilidad de aprobar la asignatura de Fisiología, se estandariza la razón de posibilidades y como vemos en la tabla 2, las variables que tienen más efecto sobre la probabilidad de aprobar la asignatura de Fisiología son las notas obtenidas en las asignaturas de Anatomía I e Histología (NotaM2 y NotaM4), debido a que son las que tienen los Odds Ratio (razón de probabilidades) estandarizados (e $e^{j} e^{j}$ ) más altos.

Tabla 2

Influencia de las variables independientes en la probabilidad de aprobar la asignatura de Fisiología. UCIMED 20082011. $(n=256)$

\begin{tabular}{lccccc}
\hline AprobM6 & $\beta$ & $\mathrm{z}$ & $\mathrm{P}>|\mathrm{z}|$ & $\theta^{\beta}$ & $\theta^{\beta}$ Estandarizado \\
\hline NotaCB2 & 0.07826 & 4.57 & 0.000 & 1.081403 & 16.716 \\
NVVM4a & 0.85242 & 2.09 & 0.037 & 2.345317 & 13.025 \\
NVVM2a & 104.698 & 2.78 & 0.005 & 2.849047 & 14.901 \\
Sexo & 0.54843 & 2.73 & 0.006 & 1.730538 & 13.092 \\
NotaM2 & 0.06182 & 4.33 & 0.000 & 1.063775 & 21.046 \\
NotaM4 & 0.08401 & 4.92 & 0.000 & 1.087642 & 20.151 \\
\hline
\end{tabular}

Nota: Elaboración propia.

\section{Discusión y conclusiones}

Diversos estudios han analizado las causas de tipo sociodemográficas y académicas de la reprobación de los cursos de los primeros semestres de las carreras de Medicina y Cirugía, en la búsqueda de soluciones que contribuyan a disminuir el problema que afecta la calidad de la educación médica (Romo y Fresán, 2001).

Al ser la reprobación un problema multifactorial, se han clasificado en - causas de origen social y familiar: desarticulación o disfuncionalidad familiar, desadaptación al medio por el origen sociocultural del que provienen, estudiantes que trabajan, problemas psicosociales y estudiantes casados o de paternidad o maternidad prematuras. - Causas de origen psicológico: desubicación en propósitos de vida e inadecuada opción vocacional. • Causas económicas: escasez de recursos y desempleo de los padres. • Causas atribuibles al rendimiento escolar: perfiles inadecuados de ingreso y falta de hábitos de estudio. • Causas físicas: problemas de salud y alimentación inadecuada. (Espinoza, 2005 citado en Nava, Rodriguez y Zambrano, 2007, p. 18). 
doi: http://dx.doi.org/10.15359/ree.19-2.5

URL: http://www.una.ac.cr/educare

CORREO: educare@una.cr

El presente estudio analiza factores de índole académico, que se sustentan en el reconocimiento de que la institución, sus estructuras formales, sus recursos y patrones de enseñanza tienen una repercusión real sobre las actuaciones del estudiantado cuando ya ha avanzado en la carrera o sea está en el transcurso de su carrera (Vélez y Roa, 2005).

Este momento es muy importante, toda vez que se conocen aquellos factores académicos que pueden incidir en su avance y, por ende, en su permanencia en la institución (Romo y Fresán, 2001).

El estudio demuestra que los factores que inciden mayormente en la posibilidad de aprobar la asignatura de Fisiología, son las calificaciones de nivel de aprobación obtenidas en los cursos previos de Anatomía I, Histología y Biología.

Esto quiere decir que, si se obtienen altas calificaciones en estas asignaturas, el estudiante tiene más probabilidad de aprobar la asignatura de Fisiología; en cambio, el repetir las asignaturas de Anatomía I e Histología, conlleva una alta posibilidad de reprobar la asignatura de Fisiología.

Lo anterior denota que en las acciones que se puedan ofrecer para pretender aminorar esta situación, se debe analizar la situación en las materias previas al curso de Fisiología, como son Anatomía I e Histología para, dentro de las posibilidades institucionales, ofertar soluciones al problema académico.

Es importante destacar que, de las variables socio demográficas analizadas (región geográfica, edad, periodo de ingreso la universidad, nacionalidad y sexo), la única que resultó significativa estadísticamente fue el sexo, que sin estandarizar, dice que ser estudiante hombre en la asignatura de Fisiología tiene un $73 \%$ más de probabilidad para aprobarla, comparado con las estudiantes mujeres. Al estandarizarla, esta magnitud tiene menos efecto sobre la probabilidad de aprobar la asignatura de Fisiología y queda en los mismos niveles de las variables, matricular más de una vez las asignaturas de Anatomía I e Histología. Este resultado concuerda con el descrito en la Universidad de Viena donde el éxito se asocia al sexo masculino (Frischenschlager, Haidinger y Mitterauer, 2005), así como a otras variables, la lengua materna y el buen desempeño académico previo. Este hallazgo es llamativo porque el proceso de feminización de las escuelas de medicina se ha ido consolidando y, en la mayoría de las universidades, este predomino ha ido acompañado de mayor éxito académico, sobre todo cuando se asocia a un rendimiento académico previo elevado. En la UCIMED, el grupo estudiantil es escogido de acuerdo con las pruebas de ingreso y sus resultados obtenidos durante la secundaria, lo cual garantiza que el grupo cuenta con buenas bases académicas. Al estar el curso de Fisiología en el IV semestre, son los cursos previos los que más podrían estar incidiendo en sus resultados, de ahí que sería necesario hacer una mayor exploración sobre estos para correlacionarlos con estos hallazgos. Sí es de indicar que el curso de Fisiología, dentro del proceso de evaluación que semestralmente se hace, siempre ha sido señalado por el grupo estudiantil como un curso de alta calidad, impartido por un grupo docente de alta calidad y con metodologías novedosas y actualizadas. 
doi: http://dx.doi.org/10.15359/ree.19-2.5

URL: http://www.una.ac.cr/educare

No obstante lo anterior, el curso de Fisiología humana, ubicado en el cuarto semestre del plan de estudios de la carrera de Medicina y Cirugía, mantiene una posición marcada por el alto índice de reprobación desde hace varias décadas. Esto ha generado que se haya analizado desde varias perspectivas y cuyos resultados han conducido a revisiones de contenido, de metodologías de enseñanza, su ubicación en el plan de estudios, requisitos, actualización de docentes en metodologías didácticas, de laboratorios y práctica, utilización de tutorías; aspectos todos que, aunque han mejorado los resultados, aún se mantienen en reprobaciones superiores al 30\%. Con este nuevo estudio se espera haber aportado más fundamentos para plantear nuevas opciones de solución a la situación planteada, manteniendo la calidad del curso, toda vez que los conocimientos y la formación en este campo componen una de las bases de la medicina.

\section{Referencias}

Ferguson, E., James, D. y Madeley, L. (Abril, 2002). Factors Associated With Success in Medical School: Systematic Review of the Literature [Los factores asociados con el éxito en la escuela de medicina: Revisión sistemática de literatura]. BMJ, 324(7343), 952-957. doi: http://dx.doi.org/10.1136/bmj.324.7343.952

Frischenschlager, O., Haidinger, G. y Mitterauer, L. (2005). Factors Associated with Academic Success at Vienna Medical School: Prospective Survey [Los factores asociados con el éxito académico en la escuela de Medicina de Viena: Encuesta prospectiva]. Croatian Medical Journal, 46(1), 58-65. Recuperado de http://www.cmj.hr/2005/46/1/15726677.htm

Hernández-Mata, J. M., Hernández-Castro, R., Nieto-Caraveo, A. y Hernández-Sierra, J. F. (Septiembre-octubre, 2005). Factores de riesgo para la deserción de estudiantes en la Facultad de Medicina de la Universidad Autónoma de San Luis Potosí (UASLP), México. Gaceta Médica de México, 141(5), 445-447. Recuperado de http://www.scielo.org.mx/pdf/ gmm/v141n5/v141n5a16.pdf

Nava, G., Rodríguez, P., y Zambrano, R. (Octubre-diciembre, 2007). Factores de reprobación en los alumnos del Centro Universitario de Ciencias de la Salud de la Universidad de Guadalajara. Revista de Educación y Desarrollo, 7, 17-25. Recuperado de http://www.cucs. udg.mx/revistas/edu desarrollo/anteriores/7/007 Nava.pdf

Ramírez, R. (2012). Cambiar, interrumpir o abandonar. La construcción de experiencias de los estudiantes en su tránsito por una institución de educación superior tecnológica (Colección Biblioteca de la Educación Superior). México: ANUIES.

Romo, A. y Fresán, M. (2001). Los factores curriculares y académicos relacionados con el abandono y el rezago (Cap. 4). En C. R. Ragueb (Comp.), Deserción, rezago y eficiencia terminal en las IES. Propuesta metodológica para el estudio (pp. 124-197). México: ANUIES. 
doi: http://dx.doi.org/10.15359/ree.19-2.5

URL: http://www.una.ac.cr/educare

CORREO: educare@una.cr

Vélez, A. y Roa, C. N. (Junio, 2005). Factores asociados al rendimiento académico en estudiantes de medicina. Educación Médica, 8(2): 74-82. Recuperado de http://scielo.isciii.es/pdf/edu/ v8n2/original1.pdf

\section{(9) Cómo citar este artículo en APA:}

Vanegas-Pissa, J. C. y Sancho-Ugalde, H. (Mayo-agosto, 2015). Identificación de factores demográficos y académicos que inciden en la aprobación de la asignatura Fisiología en la carrera de Medicina de la Universidad de Ciencias Médicas. Revista Electrónica Educare, 19(2), 83-92. doi: http://dx.doi.org/10.15359/ ree.19-2.5

Nota: Para citar este artículo en otros sistemas puede consultar el hipervínculo "Como citar el artículo" en la barra derecha de nuestro sitio web: http://www.revistas.una.ac.cr/index.php/EDUCARE/index 